\title{
Niveles de referencia de dosis para adultos en procedimientos de cardiología intervencionista en Ecuador
}

\author{
Reference even of dose for adults in procedures of cardiology interventionist in Ecuador
}

\author{
Nadia A. González-López ${ }^{*}$, Katerine M. Parra-Riofrío², Mario A. Batista-Zaldívar ${ }^{3}$, \\ Edison Carrillo-Vallejo ${ }^{4}$ y Vilma N. Yanchapanta-Bastidas ${ }^{5}$
}

${ }^{1}$ Departamento de Física, Instituto de Ciencias Básicas, Universidad Técnica de Manabí, Portoviejo; ${ }^{2}$ Hospital de Especialidades Eugenio Espejo, Pichincha, Quito; ${ }^{3}$ Departamento de Química, Instituto de Ciencias Básicas, Universidad Técnica de Manabí, Portoviejo; ${ }^{4}$ Hospital de Especialidades Carlos Andrade Marín, Pichincha, Quito; ${ }^{5}$ Facultad de Ciencias, Escuela Superior Politécnica de Chimborazo, Riobamba, Ecuador

\begin{abstract}
Resumen
Antecedentes y objetivo: El conocimiento de los niveles de referencia para estudios diagnósticos y terapéuticos es importante, dado que contribuye a la optimización de la protección radiológica de los pacientes y evita que se expongan a dosis innecesariamente altas; sin embargo, no se encontraron evidencias de estos niveles en procedimientos de cardiología intervencionista en Ecuador, por lo cual el objetivo de este estudio fue calcular los niveles de referencia diagnósticos de dosis en la superficie de entrada en pacientes adultos sometidos a procedimientos intervencionistas de cardiología (cinecoronariografía, cateterismo e intervencionismo percutáneo coronario) en la Unidad de Hemodinámica del Hospital de Especialidades Carlos Andrade Marín de Ecuador. Materiales y métodos: Las mediciones del producto dosis-área, dosis en superficie de entrada, número de imágenes y tiempo de fluoroscopia se realizaron con el angiógrafo Axiom Artis y los datos obtenidos se tabularon y procesaron con el programa informático Excel. Para este estudio se seleccionó a 145 pacientes mayores de 18 años y los valores obtenidos de niveles de referencia diagnósticos de dosis se compararon con otros informados en investigaciones anteriores y con los límites permisibles para la aparición de efectos deterministas en piel. Resultados: Este estudio permitió establecer los niveles de referencia para diagnóstico en los procedimientos intervencionistas de cardiología (cinecoronariografía, cateterismo e intervencionismo percutáneo coronario) en la Unidad de Hemodinámica del Hospital de Especialidades Carlos Andrade Marín de Ecuador. Conclusiones: Los resultados mostraron que las dosis en superficie de entrada obtenidas fueron menores a los niveles máximos recomendados por la International Atomic Energy Agency.
\end{abstract}

Palabras clave: Niveles de referencia. Dosis en superficie de entrada. Cardiología intervencionista. Efectos determinísticos. Protección radiológica.

\section{Abstract}

Antecedents and objective: The knowledge of the reference levels for diagnoses and therapeutic studies is important, because it contributes to the optimization of the radiological protection of the patients, avoiding them to be exposed to unnecessarily high doses. However, there was no evidence of these levels in interventionist cardiology procedures in Ecuador, so the objective of this study was to estimate the diagnostic reference levels of dose at the entrance surface in adult patients under-

\section{Correspondencia:}

*Nadia A. González-López

E-mail: nadiaaimegl@gmail.com
Disponible en internet: 05-01-2021 Arch Cardiol Mex. 2021;91(4):415-421 www.archivoscardiologia.com
Fecha de recepción: 02-05-2020 DOI: $10.24875 /$ ACM.20000196 CC BY-NC-ND (http://creativecommons.org/licenses/by-nc-nd/4.0/). 
going interventional cardiology procedures (cinecoronariography, catheterization and coronary percutaneous interventionism) in the Unit of Hemodynamic of the Hospital of Specialties Carlos Andrade Marín in Ecuador. Materials and methods: The measurements of product dose-area, dose in input surface, number of images and the time of fluoroscopy performed using the Axiom Artis angiographer, and the obtained data was tabulated and processed with the Excel computer program. For this study, 145 patients older than 18 years were selected, and the values obtained from diagnostic reference dose levels were compared with others reported in previous investigations and with the permissible limits for the appearance of deterministic effects on the skin. Results: This study made it possible to establish reference levels for diagnosis in interventional cardiology procedures (coronary angiography, catheterization and percutaneous coronary intervention) in the Hemodynamics Unit of the Carlos Andrade Marín Specialty Hospital in Ecuador. Conclusions: The results showed that the intake surface doses obtained were lower than the maximum levels recommended by the International Atomic Energy Agency.

Key words: Reference levels. Input surface dose. Interventional cardiology. Deterministic effects. Radiological protection.

\section{Introducción}

El uso de las radiaciones ionizantes en la medicina actual asegura el establecimiento rápido y preciso de diagnósticos radiológicos de muchas enfermedades, su tratamiento terapéutico y la vigilancia. Las técnicas empleadas para el diagnóstico tienen una alta sensibilidad, especificidad y buena reproducibilidad y son de bajo costo; los procedimientos terapéuticos pueden ser muy complejos, exigen precisión en la irradiación y algunas veces se acompañan de efectos colaterales adversos ${ }^{1}$.

La International Radiation Protection Association (IRPA) ha tratado en sus diversas publicaciones el uso de los niveles de referencia para el diagnóstico de dosis de los pacientes en la exposición médica y recomienda que cualquier exposición médica a radiaciones ionizantes debe estar justificada y optimizada, para evitar la dosis de radiación al paciente que no contribuye al propósito clínico de una tarea de diagnóstico médico por imágenes ${ }^{2-6}$.

La radiología intervencionista es una subespecialidad de la radiología en la que se utilizan técnicas de imagen para ejecutar procedimientos diagnósticos y terapéuticos guiados por fluoroscopia, ultrasonido, tomografía computarizada y resonancia magnética, cuyo objetivo principal es localizar una lesión e instituir el tratamiento adecuado ${ }^{3}$.

El United Nations Scientific Committee on the Effects of Atomic Radiation (UNSCEAR) indica que una de las fuentes principales de radiación artificial a la población mundial es la cardiología intervencionista7. Las investigaciones en el campo de las radiaciones ionizantes aplicadas a estudios diagnósticos o terapéuticos en la cardiología intervencionista están influidas por diversas variables extrañas o de intervención, razón por la cual la interpretación de sus resultados es compleja y relativa. Debido a las elevadas dosis de radiación administradas al paciente de estos procedimientos, y a su complejidad en cuanto a localización, tamaño de campo y proyecciones utilizadas, es preciso un capítulo aparte.

En virtud de lo anterior, en muchas situaciones es posible infligir en los individuos lesiones cutáneas radioinducidas graves debido a los largos tiempos de exposición, tasas de dosis altas, gran cantidad de imágenes adquiridas, inadecuada colimación y uso de filtros, entre otras razones ${ }^{8}$. Por lo tanto, es importante garantizar que las dosis de radiación recibidas por los pacientes no rebasen el umbral de aparición de los efectos deterministas (eritema dérmico: 1-24 horas después de la irradiación de 3-5 Gy; depilación: con 5 Gy es reversible, con 20 Gy es irreversible; opacidad detectable en ojo con 0.5-2 Gy y cataratas con 5 Gy) ${ }^{9}$. En la bibliografía consultada se identificaron algunos estudios sobre los niveles de referencia diagnósticos de dosis en la superficie de entrada en varios países $^{10-22}$; sin embargo, no se encontraron evidencias de éstos en procedimientos de cardiología intervencionista en la República del Ecuador.

A partir de lo anterior, el objetivo de este trabajo es calcular los niveles de referencia diagnósticos de dosis en la superficie de entrada recibida por pacientes adultos sometidos a procedimientos intervencionistas de cardiología (cinecoronariografía, cateterismo e intervencionismo percutáneo coronario) en la Unidad de Hemodinámica del Hospital de Especialidades Carlos Andrade Marín de Quito, Ecuador.

\section{Materiales y métodos}

El estudio de los niveles de referencia en procedimientos intervencionistas de cardiología (cinecoronariografía, cateterismo e intervencionismo percutáneo coronario) se realizó de acuerdo con el siguiente orden: a) análisis del estado actual de la bibliografía y del objeto de investigación, b) selección de la institución hospitalaria donde se realizaría el estudio, c) elaboración 
del procedimiento del muestreo y d) ejecución de las mediciones y procesamiento de la información.

Para el análisis del estado actual de las publicaciones y del objeto de investigación se efectuó una minuciosa revisión bibliográfica, detección y extracción de información de valor sobre el estado actual de la protección radiológica, en relación con el estudio de los niveles de referencia diagnósticos de dosis en procedimientos de cardiología intervencionista y con las recomendaciones que realizan los organismos internacionales en esta materia.

La selección de la instalación hospitalaria objeto de estudio se efectuó con base en el cumplimiento de dos condiciones: que atendiera a un elevado número de pacientes y contara con experiencia y aval técnico-profesional en la actividad de atención al paciente necesitado de procedimientos diagnósticos con imagen. En función de ello se eligió al Hospital del Instituto Ecuatoriano de Seguridad Social (IESS) Carlos Andrade Marín de Quito, que cuenta con servicios de hemodinámica en los que se realizan unos 100 procedimientos mensuales, incluidos estudios diagnósticos y terapéuticos de intervención en cardiopatías estructurales, valvuloplastias, implantes de válvulas aórticas percutáneas, implantes de stent aórticos e intervencionismo coronario.

Para la elaboración del procedimiento del muestreo, los pacientes se colocaron en decúbito supino sobre la camilla del angiógrafo, la cual tolera un peso hasta de $250 \mathrm{~kg}$, con una distancia aproximada de $1 \mathrm{~m}$ entre el tubo de rayos $X$ (debajo de la camilla) y la camilla del paciente.

En cuanto a la ejecución de las mediciones y procesamiento estadístico de la información, se siguió el procedimiento de muestreo ya explicado, se realizaron las mediciones de los niveles de referencia diagnósticos de dosis en 145 pacientes en los procedimientos de cinecoronariografía, cateterismo e intervencionismo percutáneo coronario, ya que eran los de mayor frecuencia en el servicio de hemodinámica.

El equipo utilizado para la medición fue un sistema angiográfico con intensificador de imagen (Siemens modelo AXIOM Artis) y los órganos competentes de la República del Ecuador avalaron y aprobaron su calibración.

Los datos obtenidos se agruparon en relación con los procedimientos médicos, producto dosis-área (PDA), dosis acumulada (DA) en superficie de entrada y tiempo de fluoroscopia. Además, se tomaron datos informativos de cada paciente seleccionado, como sexo, peso y talla. Se calcularon los valores medios del tiempo de fluoroscopia, los valores medios, las medianas y el cuartil $75^{\circ}$ para el PDA y la DA, para lo cual
Tabla 1. Valores de media e intervalo para tiempo de fluoroscopia

\begin{tabular}{|l|c|c|}
\hline \multirow{2}{*}{ Tipo de procedimiento } & \multicolumn{2}{|c|}{$\begin{array}{c}\text { Tiempo de exposición } \\
\text { (minutos) }\end{array}$} \\
\cline { 2 - 3 } & Media & Intervalo \\
\hline Cateterismo (diagnóstico) & 6.80 & $2.83-16.37$ \\
\hline Cinecoronariografía (diagnóstico) & 5.35 & $0.85-30.00$ \\
\hline $\begin{array}{l}\text { Intervencionismo percutáneo } \\
\text { coronario (terapéutico) }\end{array}$ & 14.58 & $4.18-42.47$ \\
\hline
\end{tabular}

Tabla 2. Valores de media, mediana y 075 para PDA según el tipo de procedimiento intervencionista

\begin{tabular}{|l|c|c|c|c|}
\hline $\begin{array}{l}\text { Tipo de } \\
\text { procedimiento }\end{array}$ & \# paciente & \multicolumn{3}{|c|}{ Producto dosis-área (Gy.cm²) } \\
\cline { 2 - 5 } & & Media & Mediana & $\mathbf{0 7 5}$ \\
\hline $\begin{array}{l}\text { Cateterismo } \\
\text { (diagnóstico) }\end{array}$ & 30 & 20.4 & 18.7 & 27.8 \\
\hline $\begin{array}{l}\text { Cinecoronariografía } \\
\text { (diagnóstico) }\end{array}$ & 65 & 33.2 & 23.2 & 42.0 \\
\hline $\begin{array}{l}\text { Intervencionismo } \\
\text { percutáneo coronario } \\
\text { (terapéutico) }\end{array}$ & 50 & 76.7 & 63.5 & 92.4 \\
\hline
\end{tabular}

se utilizó una hoja de cálculo en Excel y se tabularon para su posterior interpretación.

\section{Resultados}

Se determinaron los valores medios e intervalos para el tiempo de fluoroscopia para los procedimientos de cateterismo, cinecoronariografía e intervencionismo percutáneo coronario (Tabla 1).

Los valores medios de los tiempos de fluoroscopia para el cateterismo y cinecoronariografía fueron de 6.80 y 5.35 minutos, respectivamente, mientras que para el procedimiento de intervencionismo percutáneo coronario fue de 14.58 minutos.

La tabla 2 muestra los valores de la media, mediana y Q75 para el PDA, según corresponde a procedimientos diagnósticos y terapéuticos. Los valores de la mediana para el PDA para cateterismo y cinecoronariografía fueron de 18.7 y 23.2 Gy.cm² ${ }^{2}$, respectivamente, y para el intervencionismo percutáneo coronario de 63.5 Gy.cm².

Los valores de la media, la mediana y el Q75 para la DA en procedimientos diagnósticos y terapéuticos se muestran en la tabla 3. 
Tabla 3. Valores de media, mediana y 075 para DA según el tipo de procedimiento intervencionista

\begin{tabular}{|l|c|c|c|c|}
\hline Tipo de procedimiento & \# paciente & \multicolumn{3}{|c|}{ Dosis acumulada (mGy) } \\
\cline { 3 - 5 } & & Media & Mediana & $\mathbf{0 7 5}$ \\
\hline $\begin{array}{l}\text { Cateterismo } \\
\text { (diagnóstico) }\end{array}$ & 30 & 245.3 & 238.5 & 338.3 \\
\hline $\begin{array}{l}\text { Cinecoronariografía } \\
\text { (diagnóstico) }\end{array}$ & 65 & 552.6 & 425.0 & 682.0 \\
\hline $\begin{array}{l}\text { Intervencionismo } \\
\text { percutáneo coronario } \\
\text { (terapéutico) }\end{array}$ & 50 & 1252.0 & 995.0 & 1578.5 \\
\hline
\end{tabular}

Tabla 4. Comparación de los resultados en cuanto al tiempo de exposición de este estudio con otros informados con anterioridad en angiografía coronaria

\begin{tabular}{|c|c|c|}
\hline Autores & $\mathbf{N}$ & $\begin{array}{l}\text { Tiempo de } \\
\text { exposición } \\
\text { (minutos) }\end{array}$ \\
\hline Este estudio & 95 & 5.8 \\
\hline Jungsu, et al. $(2015)^{10}$ & 361 & 4.7 \\
\hline Humagain, Maharjan y Koju (2015) & 166 & 11.4 \\
\hline Simantirakis, et al. $(2013)^{12}$ & 2,572 & 6.0 \\
\hline Vano, et al. $(2013)^{23}$ & 1,849 & 6.5 \\
\hline Georges, et al. $(2007)^{14}$ & 3,600 & 6.3 \\
\hline Tsapaki, et al. $(2003)^{16}$ & 195 & 6.5 \\
\hline Padovani, Novario y Bernardi (1998) ${ }^{19}$ & 13 & 3.6 \\
\hline Broadhead, et al. $(1997)^{20}$ & 2,174 & 5.7 \\
\hline Zorzetto, et al. $(1997)^{21}$ & 79 & 4.9 \\
\hline
\end{tabular}

Los valores de la mediana de las DA para cateterismo y cinecoronariografía fueron de 238.5 y 425.0 mGy, respectivamente, y para el intervencionismo percutáneo coronario de $995.0 \mathrm{mGy}$.

\section{Discusión}

La determinación de los valores medios de dosis acumulada y producto dosis-área en radiología intervencionista, cualquiera que sea el procedimiento médico practicado, sea con fines diagnósticos o terapéuticos, depende de diversas variables objetivas y subjetivas, muchas veces no controlables, entre ellas protocolo clínico, edad (y peso) y equipo de rayos $X$ utilizado ${ }^{9}$.
Tabla 5. Comparación de los resultados en cuanto al tiempo de exposición de este estudio con otros informados antes en intervencionismo percutáneo coronario

\begin{tabular}{|c|c|c|}
\hline Autores & $\mathbf{N}$ & $\begin{array}{l}\text { Tiempo de } \\
\text { exposición } \\
\text { (minutos) }\end{array}$ \\
\hline Este estudio & 50 & 14.6 \\
\hline Simantirakis, et al. $(2013)^{12}$ & 1,899 & 18.0 \\
\hline Georges, et al. $(2007)^{14}$ & 3,600 & 14.0 \\
\hline Tsapaki, et al. $(2003)^{16}$ & 97 & 12.2 \\
\hline Padovani, Novario y Bernardi (1998) & 54 & 18.5 \\
\hline Broadhead, et al. $(1997)^{20}$ & 214 & 12.4 \\
\hline Zorzetto, et al. (1997) $)^{21}$ & 31 & 12.2 \\
\hline
\end{tabular}

Los tiempos de exposición a los que se sometieron los pacientes son mayores, menores o similares a otras publicaciones anteriores, según fueran los estudios documentados con los que se estableciera la comparación. Al realizar un cotejo de los resultados de este estudio en angiografía coronaria con los precedentes realizados por otros autores (tabla 4), que aparecen de forma descendente según la fecha en que se publicaron, se constató que son similares a los que notificaron Simantirakis, et al. ${ }^{12}$, Vano, et. al. ${ }^{23}$, Georges, et al. ${ }^{14}$, Tsapaki, et al. ${ }^{16}$ y Broadhead, et al..$^{20}$.

Sin embargo, hay valores medios de tiempos de exposición registrados que difieren bastante de los documentados en este estudio. Sobresalen los resultados que expusieron Humagain, Maharjan y Koju ${ }^{11}$, quienes obtienen un valor promedio de 11.4 minutos de exposición, casi el doble del alcanzado en esta investigación, y los de Padovani, Novario y Bernardi ${ }^{19}$ que registran un valor medio de 3.6 minutos, casi dos veces menor que el documentado en este estudio.

En cuanto a los valores medios de los tiempos de exposición de los pacientes en intervencionismo percutáneo coronario, los resultados de este estudio también son mayores, menores o similares de los notificados con anterioridad por otros investigadores (tabla 5).

Simantirakis, et al. ${ }^{12}$ y Padovani, Novario y Bernardi ${ }^{19}$ informaron valores similares de 18.0 y 18.5 minutos, que son mayores a los 14.6 minutos documentados en este estudio, cuya diferencia alcanza los 3.8 minutos y 4.3 minutos, respectivamente. Otros autores como Tsapaki, et al. ${ }^{16}$, Broadhead, et al. ${ }^{20}$ y Zorzetto, et al. ${ }^{21}$ también obtienen valores promedios parecidos alrededor de los 12.2 minutos y son menores a los conseguidos en 
Tabla 6. Comparación de los resultados en cuanto al PDA de este estudio con otros informados con anterioridad en angiografía coronaria

\begin{tabular}{|c|c|c|c|c|}
\hline \multirow[t]{2}{*}{ Autores } & \multirow[t]{2}{*}{$\mathbf{N}$} & \multicolumn{3}{|c|}{ Producto dosis-área (Gy.cm²) } \\
\hline & & Media & Mediana & 075 \\
\hline Este estudio & 95 & 29.1 & 21.7 & 38.1 \\
\hline Jungsu, et al. $(2015)^{10}$ & 361 & 67.6 & 54.7 & 75.6 \\
\hline Humagain, Maharjan y Koju (2015)"11 & 166 & 40.7 & & \\
\hline Simantirakis, et al. $(2013)^{12}$ & 2572 & 53.0 & & \\
\hline Vano, et al. $(2013)^{23}$ & 1849 & 32.0 & & \\
\hline D'Helft, et al. $(2009)^{13}$ & 967 & 37.9 & 30.6 & 41.7 \\
\hline Georges, et al. $(2007)^{14}$ & 3600 & 63.0 & & \\
\hline Sapiin, Ng y Abdullah (2004) ${ }^{15}$ & 176 & 48.6 & 37.0 & 59.6 \\
\hline Tsapaki, et al. $(2003)^{16}$ & 195 & 47.3 & 39.1 & 60.4 \\
\hline Van de Putte, et al. $(2000)^{17}$ & 62 & 60.6 & 56.8 & 80.6 \\
\hline Bestou, et al. $(1998)^{18}$ & 29 & 30.4 & & \\
\hline Padovani, Novario y Bernardi (1998) & 13 & 39.3 & & \\
\hline Broadhead, et al. $(1997)^{20}$ & 2174 & 57.8 & 45.5 & 69.9 \\
\hline Zorzetto, et al. $(1997)^{21}$ & 79 & 55.9 & 52.5 & 65.6 \\
\hline Vano, et al. $(1995)^{22}$ & 288 & 66.5 & 45.7 & 69.3 \\
\hline
\end{tabular}

esta investigación, pero sólo por 2.2 o 2.4 minutos de diferencia. En cambio, Georges, et al..$^{14}$ con 14.0 minutos muestra resultados similares a los alcanzados en este protocolo.

Al establecer una comparación entre el PDA obtenido en la angiografía coronaria en esta investigación con otros 14 estudios documentados en las publicaciones médicas consultadas (tabla 6) se confirmó que todos ellos señalan valores de media, mediana y percentil $75^{\circ}$ superiores. Sólo en la media del PDA, los estudios realizados de Vano, et. al. ${ }^{23}$ y Bestou, et al. ${ }^{18}$ registraron un valor similar.

La media obtenida en el PDA en la angiografía coronaria en este estudio (29.1 Gy.cm²) es semejante a la que recomienda la International Atomic Energy Agency ${ }^{24}$, que sugiere un valor medio de $26.0 \mathrm{~Gy} . \mathrm{cm}^{2}$.

En la comparación efectuada del PDA obtenido en el intervencionismo percutáneo coronario en este estudio con otras 11 investigaciones documentadas en las publicaciones consultadas (tabla 7) se observó que seis de ellas ${ }^{13,16,18,20-22}$ notifican valores de la media, la mediana y el percentil $75^{\circ}$ similares. Es necesario destacar que estas indagaciones se condujeron en un periodo de 14 años y en contextos diferentes.
Simantirakis, et al. ${ }^{12}$, Georges, et al. ${ }^{14}$, Sapiin, $\mathrm{Ng}$ y Abdullah ${ }^{15}$, Van de Putte, et al. ${ }^{17}$ y Padovani, Novario y Bernardi ${ }^{19}$ informan medias mayores a 76.7 Gy.cm ${ }^{2}$ documentadas en este estudio, cuyas diferencias alcanzan valores superiores a los $23.0 \mathrm{~Gy} . \mathrm{cm}^{2}$. Algo semejante ocurre con la mediana y el Q75.

La media obtenida en el PDA para el intervencionismo percutáneo coronario (76.7 Gy.cm²) es mayor que la recomendada por la International Atomic Energy

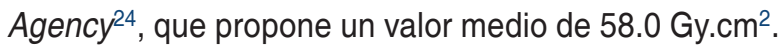
Es importante señalar que todos los estudios similares consultados (tabla 7) señalan valores superiores a los sugeridos por esta institución.

Sin embargo, como resultado del proyecto Measures for optimising radiological information and dose in digital imaging and interventional radiology, financiado por la Comisión Europea, se han publicado NRD preliminares para los procedimientos cardiológicos: angiografía coronaria y angioplastia transluminal percutánea coronaria de 66.5 y $87.5 \mathrm{~Gy}_{\mathrm{cm}}{ }^{2}$, respectivamente ${ }^{9}$, que son mayores a los que propone la International Atomic Energy Agency ${ }^{24}$ para ambos casos. Esto confirma la relatividad de los estudios relacionados con esta temática. 
Tabla 7. Comparación de los resultados en cuanto al PDA de este estudio con otros notificados antes en intervencionismo percutáneo coronario

\begin{tabular}{|c|c|c|c|c|}
\hline \multirow[t]{2}{*}{ Autores } & \multirow[t]{2}{*}{$\mathbf{N}$} & \multicolumn{3}{|c|}{$\begin{array}{l}\text { Producto dosis-área } \\
\qquad\left(\mathrm{Gy}, \mathrm{cm}^{2}\right)\end{array}$} \\
\hline & & Media & Mediana & 075 \\
\hline Este estudio & 50 & 76.7 & 63.4 & 92.4 \\
\hline Simantirakis, et al. $(2013)^{12}$ & 1,899 & 129.0 & & \\
\hline $\mathrm{D}^{\prime} \mathrm{Helft}$, et al. $(2009)^{13}$ & 463 & 78.3 & 58.1 & 83.6 \\
\hline Georges, et al. $(2007)^{14}$ & 3,600 & 100.0 & & \\
\hline Sapiin, $\mathrm{Ng}$ y Abdullah $(2004)^{15}$ & 70 & 153.0 & 103.0 & 189.5 \\
\hline Tsapaki, et al. $(2003)^{16}$ & 97 & 68.0 & 58.3 & 80.7 \\
\hline Van de Putte, et al. $(2000)^{17}$ & 10 & 165.9 & 131.6 & 185.8 \\
\hline Bestou, et al. $(1998)^{18}$ & 7 & 70.7 & & \\
\hline $\begin{array}{l}\text { Padovani, Novario y Bernardi } \\
(1998)^{19}\end{array}$ & 54 & 101.9 & & \\
\hline Broadhead, et al. $(1997)^{20}$ & 214 & 77.9 & 61.1 & 100.6 \\
\hline Zorzetto, et al. $(1997)^{21}$ & 31 & 91.8 & 82.6 & 104.6 \\
\hline Vano, et al. $(1995)^{22}$ & 45 & 87.5 & 66.7 & 122.3 \\
\hline
\end{tabular}

Según lo planteado por la International Commission on Radiological Protection ${ }^{4}$, en la publicación 85 , el umbral de dosis en piel para la aparición de efectos determinísticos corresponde a 2000 mGy. En este estudio, los valores de dosis acumulada (DA) fueron inferiores para los procedimientos de cardiología intervencionista, sea diagnóstica o terapéutica.

Los valores del percentil $75^{\circ}$ de PDA y DA para los procedimientos intervencionistas diagnósticos obtenidos en este estudio son menores a los comunicados ${ }^{6,24,25}$; sin embargo, para el procedimiento terapéutico de intervencionismo percutáneo coronario son mayores.

\section{Conclusiones}

Los tiempos de exposición medidos en este estudio, tanto en los procedimientos de angiografía coronaria como en el intervencionismo percutáneo coronario, son mayores, menores o similares a los obtenidos por otras publicaciones anteriores; no obstante, hay valores medios registrados que difieren demasiado de los documentados en este estudio.

Los valores de media, mediana y percentil $75^{\circ}$ del PDA obtenido en angiografía coronaria en esta investigación son menores a los notificados en otros 13 estudios documentados en las publicaciones consultadas y la media lograda en este estudio (29.1 Gy.cm²) es similar a la que recomienda la International Atomic Energy Agency ${ }^{24}$ (26.0 Gy.cm²).

Los valores de media, mediana y percentil $75^{\circ}$ del PDA obtenido en el intervencionismo percutáneo coronario en este estudio son mayores o semejantes a los informados en otras 11 investigaciones documentadas en las publicaciones consultadas y la media alcanzada en el PDA (76.7 Gy.cm²) es mayor que la recomendada por la Inter-

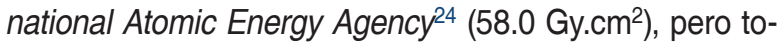
dos los estudios similares consultados señalan también valores superiores a los sugeridos por este organismo.

Los valores de dosis acumulada obtenidos para los procedimientos de cardiología intervencionista, tanto diagnóstica como terapéutica, son inferiores al umbral de dosis en piel de 2000 mGy que plantea la International Commission on Radiological Protection 4 para la aparición de efectos determinísticos.

En virtud de la variabilidad que muestran los resultados de los tiempos de exposición, el PDA y la DA para procedimientos cardiológicos diagnósticos y terapéuticos en estudios de cardiología intervencionista, deben realizarse investigaciones similares en otras instituciones hospitalarias del país y otros estudios para reconocer las posibles correlaciones de estas variables con la edad, peso y talla por grupos de pacientes, la aparición de efectos deterministas en piel, además de otras variables como dosis por minuto de exposición (mGy/min), número de imágenes por minuto de exposición (\# imágenes/min) y minutos de exposición por imágenes tomadas (min/imagen).

\section{Agradecimientos}

Los autores de esta investigación desean agradecer a las autoridades de la Universidad Técnica de Manabí, del Hospital del IESS Carlos Andrade Marín y la Escuela Superior Politécnica de Chimborazo por su colaboración para la realización de ésta, así como al Proyecto "Establecimiento de niveles referenciales diagnóstico en procedimientos de intervencionismo cardiológico adulto en América Latina", y a su director Carlos Ubeda de la Cerda de la Universidad de Tarapaca de Chile.

\section{Financiamiento}

Esta investigación no ha recibido ninguna beca específica de agencias de los sectores público, comercial o sin ánimo de lucro. 


\section{Conflicto de intereses}

Los autores declaran no tener ningún conflicto de intereses

\section{Responsabilidades éticas}

Protección de personas y animales. Los autores declaran que para esta investigación no se han realizado experimentos en seres humanos ni en animales.

Confidencialidad de los datos. Los autores declaran que han seguido los protocolos de su centro de trabajo sobre la publicación de datos de pacientes.

Derecho a la privacidad y consentimiento informado. Los autores declaran que en este artículo no aparecen datos de pacientes.

\section{Bibliografía}

1. Instituto de Tecnología Nuclear Dan Beninson. La radiación y su paciente: una guía para médicos. Argentina: Instituto de Tecnología Nuclear Dan Beninson, 2009:40.

2. International Commission on Radiological Protection (ICRP). Recommendations of the International Commission on Radiological Protection. Publication 103. Madrid: Senda, 2007.

3. International Commission on Radiological Protection (ICRP). Radiation and your patient: a guide for medical practitioners. ICRP 21. Madrid: Senda, 2002:39-47.

4. International Commission on Radiological Protection (ICRP). Avoidance of radiation injuries from medical interventional procedures. Publication 85. Madrid: Senda, 2000:7-37.

5. International Commission on Radiological Protection (ICRP). Radiological protection and safety in medicine. Publication 73. ICRP 26(2). Madrid: Senda, 1996.

6. International Commission on Radiological Protection (ICRP). Recommendations of the International Commission on Radiological Protection. Publication 60. ICRP 21(1-3). Madrid: Senda, 1991.

7. Corredoira E. Dosimetría a los pacientes pediátricos en cateterismos cardíacos e impacto de las adquisiciones en modo tomográfico [Tesis]. [PDF]. Universidad Complutense de Madrid, España. 2016:185. Consultado el 6 de enero de 2018. Disponible en: https://eprints.ucm.es/38888/1/ T37687.pdf.
8. Canevaro L. Aspectos físicos e técnicos da radiología intervencionista. Rev Bras Fís Méd. 2009;3(1):101-15.

9. Ruiz P. Procedimientos de dosimetría para pacientes en radiodiagnóstico. En: Brosed A (editor). Fundamentos de física médica. Vol. 2. Sociedad Española de Física Médica. España: 2012:247-334.

10. Jungsu K, Deoknam S, Inseok C, Sora N, Yongsu Y, Hyunji K, et al. Development of diagnostic reference levels using a real-time radiation dose monitoring system at a cardiovascular center in Korea. J Digit Imaging. 2015;28(6):684-94.

11. Humagain S, Maharjan R, Koju R. Radiation exposure to the patient during diagnostic coronary angiogram at Dhulikhel Hospital. Kathmandu Univ Med J. 2015;13(1):61-3.

12. Simantirakis G, Koukorava C, Kalathaki M, Pafilis C, Kaisas I, Economides S, et al. Reference levels and patient doses in interventional cardiology procedures in Greece. Eur Radiol. 2013;23(8):2324-32.

13. D'Helft CJ, Brennan PC, Mcgee AM, Mcfadden SL, Hughes CM, Winder RJ, et al. Potential Irish dose reference levels for cardiac interventional examinations. Br J Radiol. 2009;82(976):296-302.

14. Georges JL, Livarek B, Gibault-Genty G, Messaoudi H, Aziza JP, Hautecoeur JL, et al. Variatins of radiatin dosage delivered to patints undergoing interventinal cardiological procedures. A monocentric study 2002-05. Archives Des Maladies Du Et Des Coeur Vaisseaux. 2007;100(3):175-81.

15. Sapiin $\mathrm{B}, \mathrm{Ng} \mathrm{KH}$, Abdullah JJ. Radiation dose to patients undergoing interventional radiological procedure in selected hospital in Malaysia: retrospective study. Hong Kong J Radiol. 2004;7(3):129-36.

16. Tsapaki V, Kottou S, Vano E, Faulkner K, Giannouleas J, Padovani R, et al. Patient dose values in a dedicated Greek cardiac centre. $\mathrm{Br} J$ Radiol. 2003;76(910):726-30.

17. Van de Putte S, Verhaegen F, Taeymans $Y$, Thierens $H$. Correlation of patient skin doses in cardiac interventional radiology with dose area product. Br J Radiol. 2000;73(869):504-13.

18. Bestou S, Efstathopoulos EP, Katritsis D, Faulkner K, Panayiotakis G Patient radiation doses during cardiac catheterization procedures. $\mathrm{Br} \mathrm{J}$ Radiol. 1998;71(846):634-9.

19. Padovani $R$, Novario $R$, Bernardi $G$. Optimization in coronary angiography and percutaneous transluminal coronary angioplasty. Radiat Prot Dosim. 1998;80(1-3):303-6.

20. Broadhead DA, Chapple CL, Faulkner K, Davies ML, McCallum H. The impact of cardiology on the collective effective dose in the North of England. Br J Radiol. 1997;70(833):492-7.

21. Zorzetto M, Bernardi G, Morocutti G, Fontanelli A. Radiation exposure to patients and operators during diagnostic catheterization and coronary angioplasty. Catheter Cardiovasc Interv. 1997;40(4):348-51.

22. Vano E, González L, Fernández JM, Guibelalde E. Patient dose values in interventional radiology. Br J Radiol. 1995;68(815):1215-20.

23. Vano E, Fernández JM, Sánchez RM, Ten JI. Niveles de referencia de dosis en radiología intervencionista. Radiología. 2013;55(S2):17-24.

24. International Atomic Energy Agency (IAEA). Protección radiológica de los pacientes en cardiología intervencionista. Vienna International Centre. Austria. 2013. Consultado el 23 de marzo de 2019. Disponible en: https:// rpop.iaea.org/RPOP/RPoP/Content-es/InformationFor/HealthProfessionals/5_ Interventional Cardiology/index.htm\#ref11.

25. International Atomic Energy Agency (IAEA). Patient dose optimization in fluoroscopically guided interventional procedures. Final report of a coordinated research Project. Vienna International Centre. Austria. 2010:110. 